\title{
Biomimetic Biodegradable Artificial Antigen Presenting Cells Synergize with PD-1 Blockade to Treat Melanoma
}

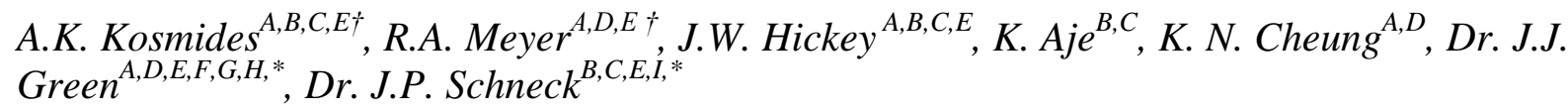

${ }^{\mathrm{A}}$ Department of Biomedical Engineering, ${ }^{\mathrm{B}}$ Institute for Cell Engineering, ${ }^{\mathrm{C}}$ Department of Pathology, ${ }^{\mathrm{D}}$ Translational Tissue Engineering Center, ${ }^{\mathrm{E}}$ Institute for Nanobiotechnology, ${ }^{\mathrm{F}}$ Department of Materials Science and Engineering, ${ }^{\mathrm{G}}$ Department of Ophthalmology, Department of Oncology, ${ }^{\mathrm{I}}$ Department of Medicine, Johns Hopkins University School of Medicine, Baltimore, MD, 21231, USA.

$\dagger$ These authors contributed equally to this work.

* Corresponding authors, email: green@jhu.edu, jschnec1@jhmi.edu

Keywords: artificial antigen presenting cell, immunotherapy, CD8+ T-cell, PD-1, combination therapy

\section{Introduction}

Biomimetic materials that target the immune system hold promise for cancer immunotherapy [1]. Synthetic immunotherapies can be designed with defined characteristics and therefore often outperform their cell-based counterparts. These platforms can be engineered in terms of biodegradability [2], controlled release of immuno-modulators [3], and physical parameters including shape and size [4]. Biomimetic materials can be customized to incorporate combination therapies in an all-in-one therapeutic and are therefore an exciting platform for the future of cancer immunotherapy. Despite their potential, current development of combinatorial immunotherapies utilizing biomaterials has been limited as their interaction with other existing therapeutics must first be understood.

Synthetic artificial antigen presenting cells (aAPC), a biomaterial-based immunotherapy, have shown success in generating an anti-tumor immune response in vitro and in vivo [5-8]. aAPC are three-dimensional platforms that minimally express the two signals required for $\mathrm{T}$ cell activation - a signal 1, peptide-MHC ( $\mathrm{pMHC}$ ) to provide $\mathrm{T}$ cell receptor (TCR) specificity, and a signal 2, such as anti-CD28 monoclonal antibody (mAb) to provide the co-stimulatory "go" signal. aAPC can be functionalized with tumor-specific pMHC to activate a patient's immune system against cancer antigens and mediate tumor 
rejection [9-11]. They can be utilized in adoptive cell transfer (ACT) of ex vivo activated autologous T cells $[9,12,13]$ or directly administered intravenously (IV) for in vivo anti-tumor T cell activation $[14,15]$. Synthetic aAPC platforms have distinct advantages over cellular systems in terms of long-term storage and the ability to optimize T cell activation and biocompatibility [16]. Unlike biological antigen presenting cells used as cellular therapy, biomaterial-based aAPC have the advantage of being able to maintain an "always on" state that cannot be down-regulated by the microenvironment as well as flexibility for manufacturing as an acellular product. Compared to PLGA-based drug delivery particles for cancer therapy, the anti-cancer drugs must reach and destroy every cancer cell to ultimately be effective. In contrast, PLGA-based aAPC particles for immunotherapy need only reach tumor specific $\mathrm{T}$ cells that can recognize the tumor antigen for the aAPC to then be able to direct a robust systemic immunotherapy response against the cancer cells. Biomimetic modifications of PLGA-based aAPC materials that greatly enhance their effector capacity, including controlling the shape of the aAPC $[4,17]$ or slowly releasing pro-inflammatory cytokines from their core $[18,19]$, have demonstrated the benefit of bringing novel materials engineering concepts to the development of immunotherapeutics.

In addition to amplifying positive regulators of the immune system, inhibiting negative regulators has also shown success in generating anti-tumor immune responses. Checkpoint molecules, including programmed death 1 (PD-1) and CTLA-4, are negative regulators of $\mathrm{T}$ cell function. These molecules are upregulated on tumor infiltrating lymphocytes and on activated T cells expanded during ACT, being described as a rheostat of the immune system [20]. PD-1 signaling inhibits CD8+ T cell effector function upon ligation with its ligand, programmed death ligand 1 (PD-L1), and is one of the methods by which tumors escape immune surveillance. Checkpoint blockade with monoclonal antibodies against PD-1 and PD-L1 delay tumor growth in murine tumor models [21,22], and FDA approved monoclonal anti-PD-1 and anti-CTLA-4 antibodies have shown significant overall response 
rates and long-term survival benefits. However, clinical responses only reach approximately $30 \%$ [23-26] indicating that there is a necessity for improvement.

Single-targeted approaches have limited efficacy because cancerous cells utilize multiple mechanisms to avoid immune surveillance and the immune system internally suppresses prolonged strong activation [27]. The combination of checkpoint inhibitors with other immunotherapies that boost $\mathrm{T}$ cell effector functions or promote cancer cell recognition by the immune system have potential to increase anti-tumor effectiveness. Checkpoint blockade in conjunction with $\mathrm{T}$ cell costimulatory antibodies resulted in tumor regression in multiple murine tumor models [28-30] and increased effector functions of exhausted CD8+ T cells by forcing them out of quiescence [31]. These studies suggest that checkpoint blockade can boost the effects of other immune-stimulatory approaches, although their interaction with biomaterial-based antigen-specific $\mathrm{T}$ cell stimulation has not been studied.

Here, we investigate the synergy between a biomimetic material, biodegradable PLGA-based aAPC, and anti-PD-1 monoclonal antibody treatment for the activation of tumor-specific CD8+ T cells. Combinatorial treatment enhances CD8+ T cell effector functions in vitro and significantly delays tumor growth in vivo. These results demonstrate the effectiveness of PLGA-based aAPC in combination immunotherapy, and identify a molecule that could potentially be incorporated and released from polymeric aAPC for increased effectiveness.

\section{Materials and methods}

\section{1. Artificial antigen presenting cell synthesis and characterization}

Artificial antigen presenting cells were synthesized in a two-step core particle formation and functionalization. Particles cores were synthesized from poly (lactic-coglycolic acid) (PLGA 50:50 lactic acid to glycolic acid ratio, MW 34,000-58,000 Da) that was purchased commercially (Sigma Aldrich; St. Louis, MO). For a typical microparticle 
synthesis, $100 \mathrm{mg}$ of PLGA was dissolved in $5 \mathrm{~mL}$ dichloromethane and homogenized into a $50 \mathrm{~mL}, 1 \%$ poly vinyl alcohol (PVA) solution by an T-25 digital ULTRA-TURRAX IKA tissue homogenizer at a speed of 5,000 rpm (IKA Works; Wilmington, NC). The resulting microparticle emulsification was then added to $100 \mathrm{~mL}$ of $0.5 \%$ PVA solution. The dichloromethane was then allowed to evaporate over the course of 4 hrs. After particle hardening, the particles were washed three times in water through centrifugation at $3000 \mathrm{~g}$ for 5 min. The washed microparticle solution was flash frozen in liquid nitrogen and lyophilized for 1 day prior to characterization and use.

Functionalization was achieved through EDC/NHS chemistry to conjugate carboxylic acid terminated PLGA to amines on the proteins of interest. Lyophilized particles were dissolved in $0.1 \mathrm{M}$ MES buffer at $\mathrm{pH} 6.0$ at a concentration of $2 \mathrm{mg} / \mathrm{mL} .100 \mu \mathrm{L}$ of EDC/NHS (Sigma Aldrich; St. Louis, MO) stock solution at $40 \mathrm{mg} / \mathrm{mL}$ and $48 \mathrm{mg} / \mathrm{mL}$ respectively were added to each sample and the particles were activated for $30 \mathrm{~min}$. The resulting surface activated particles were washed in PBS through centrifugation at 5,000 $\mathrm{g}$ for 5 min. The particles were resuspended in PBS at $2 \mathrm{mg} / \mathrm{mL} .8 \mu \mathrm{g}$ MHC IgG dimer loaded with the antigen of choice and $10 \mu \mathrm{g}$ anti-CD28 monoclonal antibody (mAb) (BD Biosciences; San Jose, CA) was added to each sample and the particles were allowed to react with the proteins overnight at $4{ }^{\circ} \mathrm{C}$. The resulting aAPC were washed $3 \mathrm{x}$ in $\mathrm{PBS}$ through centrifugation at 5,000 $\mathrm{g}$ and then dissolved in $400 \mu \mathrm{L}$ of $100 \mathrm{mg} / \mathrm{mL}$ endotoxin free sucrose. The resulting suspension was then lyophilized overnight.

Particle imaging was conducted with a Leo FESEM scanning electron microscope. To prepare samples for analysis, lyophilized particles were mounted onto carbon tape (Nisshin EM Co.; Tokyo, Japan) and placed upon aluminum tacks (Electron Microscopy Services; Hatfield, PA). The excess particles were removed by blowing air across the surface of the tack and the sample was then sputter coated with a $20 \mathrm{~nm}$ thick layer of gold-palladium. The 
samples were then loaded into the microscope and imaged. The images were processed in ImageJ to obtain size information.

To determine the amount of protein on the surface, conjugated aAPC microparticles were stained with Alexa Fluor 647 goat anti-mouse IgG for the dimer and Alexa Fluor 546 goat anti-hamster IgG for the anti-CD28 (Life Technologies; Grand Island, NY) for 1 hour at $4{ }^{\circ} \mathrm{C}$. The particles were subsequently washed with PBS three times and fluorescence readings of particles were evaluated for fluorescence with a BioTek Synergy 2 plate reader (Biotek; Winooski, VT). The mass of protein on the particle was calculated to evaluate conjugation efficiency. Conjugation efficiency was calculated as (Protein Calculated on Particles)/(Protein Added to Conjugation Media) $* 100 \%$.

\subsection{Anti-PD1 monoclonal antibody synthesis}

Anti-PD-1 mAb clone G4 was grown from the G4 hybridoma cell line. Hybridoma cells were grown in hybridoma serum free media supplemented with L-glutamine. After one week, the supernatant was harvested and run over a HiTrap protein G column (GE Healthcare, Little Chalfont, Buckinghamshire, UK), then eluted according to the manufacturer's protocol. G4 mAb was concentrated by membrane ultrafiltration with a Vivaspin $2050 \mathrm{kDa}$ MWCO (GE Healthcare) and concentration was measured by Nanodrop ND-1000 Spectrophotometer.

\subsection{In vitro artificial antigen presenting cell $\mathrm{T}$ cell stimulation}

To determine the effectiveness of the aAPC at stimulating antigen specific T cells, we used primary CD8+ T cells isolated from PMEL or $2 \mathrm{C}$ mouse splenocytes. All mice were maintained according to Johns Hopkins University's Institutional Review Board. The mice were sacrificed and then the spleen was dissected out and homogenized through a cell strainer. The CD8+ T cells were then isolated using the Miltenyi CD8a+ Isolation Kit (Miltenyi; Auburn, CA). The cells were then stained with Vybrant Cell Tracker carboxyfluorescein 
succinyl ester (CFSE) (Life Technologies; Grand Island, NY) dye following the manufacturer's protocol. CFSE stained cells were incubated with the particle bearing the antigen of choice at a concentration of $1 \mathrm{mg}, 0.1 \mathrm{mg}$, or $0.01 \mathrm{mg}$ aAPC (polymer weight)/100,000 CD8+ T cells in RPMI supplemented with L-glutamine, non-essential amino acids, vitamin solution, sodium pyruvate, $\beta$-mercaptoethanol, $10 \%$ FBS, ciproflaxin, and a cocktail of T cell growth factors. CFSE dilution was then assessed after three days of incubation through flow cytometry analysis on a BD FACSCalibur. Each generation is defined as a distinct peak of the flow cytometry CFSE histogram, as the CFSE dye is diluted in half with each cell division. Generational analysis was assessed using the built in function in FlowJo (TreeStar). Total proliferation after seven days was assessed by cell counting on a hemocytometer and using a trypan blue exclusion test to exclude dead cells.

\subsection{In vitro anti-PD-1 mAb functionality assay}

To evaluate the functional effectiveness of the synthesized anti-PD-1 mAb, we utilized an in vitro assay of repeated CD8+ T cell stimulation to upregulate PD-1 expression, as PD-1 expression is low on naïve CD8+ T cells. On day 0 , primary splenocytes were isolated from naïve 2C transgenic mouse (Jackson Labs; Bar Harbor, ME) spleens through cell straining. Cells were treated with $4 \mathrm{~mL}$ of ACK lysis buffer for 1 minute to lyse red blood cells. CD8+ T cells were isolated by negative selection with the Miltenyi CD8a+ Isolation Kit following the manufacturer's protocol (Miltenyi; Auburn, CA). Micro anti-CD3 (145.2C11)/anti-CD28 (37.51) aAPC were synthesized on $4.5 \mu \mathrm{m}$ M-450 Epoxy Dynabeads (Life Technologies; Grand Island, NY) at a 1:1 protein ratio, following manufacturer's protocol. Anti-CD3 and anti-CD28 antibodies were purchased from BioXCell (West Lebanon, NH). 2C CD8+ T cells were mixed with micro aAPC at a 1:1 ratio and cultured in RPMI supplemented with Lglutamine, non-essential amino acids, vitamin solution, sodium pyruvate, $\beta$-mercaptoethanol, $10 \%$ FBS, ciproflaxin, and a cocktail of T cell growth factors. On day 4, additional T cell 
growth factors and anti-CD3/anti-CD28 beads were added at a 2:1 bead:cell ratio. On day 6, B16-SIY and B16-F10 cells were cultured in RPMI supplemented with L-glutamine, nonessential amino acids, vitamin solution, sodium pyruvate, $\beta$-mercaptoethanol, $10 \%$ FBS, ciproflaxin, and $20 \mathrm{ng} / \mathrm{ml}$ recombinant murine IFN- $\gamma$ (R\&D Systems, Minneapolis, MN) to upregulate PD-L1 expression.

On day 8, B16-F10 and B16-SIY were harvested and washed three times to remove all IFN- $\gamma$, as confirmed by ELISA. CD8+ T cells were also harvested, washed three times, and aAPC were removed with a magnet. PD-1 and PD-L1 expression was confirmed on CD8 and B16 cells by flow cytometry using fluorescently labeled anti-PD-1 and anti-PD-L1 antibodies (Biolegend). CD8+ T cells and B16-SIY or B16-F10 cells were mixed at a 1:1 effector target ratio in the presence of $10 \mu \mathrm{g} / \mathrm{ml}$ anti-PD-1 mAb or Armenian hamster IgG isotype control antibody. The cells were co-incubated for 18 hours at $37^{\circ} \mathrm{C}$, then supernatants were collected. IFN- $\gamma$ was measured by ELISA using the ebioscience murine IFN- $\gamma$ Ready-SET-Go! Kit (San Diego, CA).

\subsection{In vitro anti-PD-1 mAb and aAPC assay}

To evaluate the synergistic effect of aAPC and anti-PD-1 mAb treatment, we utilized an in vitro $\mathrm{T}$ cell and tumor cell co-incubation assay. CD8+ $\mathrm{T}$ cells were stimulated at a single earlier time-point prior to the start of the assay to allow for resting time before the secondary stimulation. On day -5 , primary splenocytes were isolated from naïve $2 \mathrm{C}$ transgenic mouse (Jackson Labs; Bar Harbor, ME) spleens through cell straining, and incubated with a 1:1 ratio of anti-CD3/anti-CD28 microbeads as above. Additional T cell growth factors and media were added on day -2. On day -2, B16-SIY and B16-F10 cells were cultured in the presence of $20 \mathrm{ng} / \mathrm{ml}$ IFN- $\gamma$, as above. On day 0, B16-F10 and B16-SIY were harvested and washed three times to remove all IFN- $\gamma$, as confirmed by ELISA. 2C cells were also harvested, washed three times, and aAPC were removed with a magnet. Cells were stained with PE anti- 
PD-1, PE anti-PD-L1, PE anti-PD-L2, and isotype control antibody (Biolegend) and read on a BD FacsCalibur to confirm expression. CD8+ T cells and B16-SIY or B16-F10 cells were mixed at a 1:1 effector target ratio, and anti-PD-1 mAb and PLGA aAPC were added into culture at titrating amounts. The cells were incubated for 18 hours at $37^{\circ}$, then supernatants were collected. IFN- $\gamma$ was measured by ELISA using the ebioscience murine IFN- $\gamma$ ReadySET-Go! Kit (San Diego, CA).

\subsection{In vivo particle and cell biodistribution study}

To evaluate the influence of the combination of adoptively transferred cells on the biodistribution of our aAPC, we used near infrared (IR) fluorescence to track the aAPC upon intravenous administration. The particles and aAPC were synthesized as previously described except that $1 \mathrm{mg}$ of a custom synthesized hydrophobic dye from LI-COR biotechnologies fluorophore (LICOR Biosciences; Lincoln, NE) was added to the dichloromethane mixture to be encapsulated into the particles. Labeled aAPC were split up into two different treatment groups. For group 1, Thy 1.2+ C57BL/6 mice (Jackson Laboratories; Bar Harbor, ME) received intravenously $2 \mathrm{mg}$ of the IR labeled aAPC alone. For group 2, Thy $1.2+$ C57BL/6 mice received intravenously $2 \mathrm{mg}$ of IR labeled aAPC with $1 \times 10^{6}$ Thy $1.1+$ PMEL CD8+ T cells that had been pre-incubated for 1 hour at $4{ }^{\circ} \mathrm{C}$. Blood was collected retroorbitally at 10 , 20, 30, and 40 min post injection to monitor elimination from the bloodstream and was imaged in the LI-COR Pearl Impulse. At 24 hours, mice were sacrificed and the liver, kidney, spleen, heart, and lung were dissected out and imaged in the LI-COR Pearl Impulse (LICOR Biosciences; Lincoln, NE) to determine biodistribution of the particles over $24 \mathrm{hrs.} \mathrm{All}$ fluorescence was quantified by ImageJ for normalized measurements of particle concentration. For retroorbital bleeds, the data for each mouse was normalized to the fluorescence value of the initial time point collected. This data was then fit to a single exponential decay curve using the GraphPad non-linear regression analysis module (GraphPad Software; La Jolla, CA). 
For organ distribution, the fluorescence readings were normalized to the sum of the fluorescence values across all organs to get a percent distribution across the organs analyzed.

\subsection{In vivo tumor treatment study}

To evaluate the efficacy of dual treatment in vivo we utilized an adoptive immunotherapy murine melanoma treatment model. Power calculations were performed to determine the necessary group size using assumptions based on previous experience with subcutaneous B16-F10 tumor models. For a two-sided t-test at power level 0.8 and significance level 0.05 , at least 6 mice are needed for each group to see statistically significant data in tumor burden differences.

Thy 1.2+ C57BL/6 mice (Jackson Laboratories; Bar Harbor, ME) were inoculated subcutaneously on the right flank with $3 \times 10^{5}$ B16-F10 melanoma cells four days prior to treatment. One day prior to treatment, the mice were irradiated with a central dose of 500 cGy, a sublethal dose to induce transient lymphopenia as per standard approaches to adoptive immunotherapy [32]. On the day of treatment, when tumors were palpable, mice were subdivided into four groups randomly by cage: 1) no treatment, 2) CD8 adoptive transfer + anti-PD-1 mAb, 3) CD8 adoptive transfer + aAPC, or 4) CD8+ T cell adoptive transfer + antiPD-1 mAb + aAPC. All injection volumes were $100 \mu \mathrm{L}$. All injections were completed intravenously, and group 1 received mock injections of PBS, labeled as no treatment control. Group 2 received an injection of $1 \times 10^{6}$ Thy $1.1+$ PMEL CD8+ T cells and $200 \mu \mathrm{g}$ of anti-PD1 antibody intraperitoneally. Group 3 received an injection of $1 \times 10^{6}$ Thy $1.1+$ PMEL CD8+ T cells that had been pre-incubated with $2 \mathrm{mg}$ of PLGA aAPC particles for one hour at $4^{\circ} \mathrm{C}$. Group 4 received an injection of $1 \times 10^{6}$ Thy $1.1+$ PMEL CD8+ T cells that had been preincubated with $2 \mathrm{mg}$ of PLGA aAPC particles as well as $200 \mu \mathrm{g}$ of anti-PD-1 antibody intraperatoneally. One day post treatment, Groups 2 and 4 received an additional $100 \mu \mathrm{g}$ of anti-PD-1 antibody intraperitoneally. PD-1 expression on PMEL cells stimulated with 1 
$\mathrm{mg} / \mathrm{ml}$ aAPC was confirmed by staining with an APC-anti-CD8 and PE-anti-PD-1 or isotype antibody (Biolegend) and reading them by flow cytometry after 7 and 24 hours.

At six, ten, and thirteen days post treatment, the mice were bled retroorbitally to analyze peripheral blood. Red blood cells were lysed with 4 min of treatment in ACK lysis buffer and then the remaining white blood cells were stained with anti-CD8a-APC (BD Biosciences; San Jose, CA) and anti-Thy1.1-Alexa Fluor 488 (BioLegend). The cells were then analyzed by flow cytometry on a BD FACSCalibur to evaluate the percentage of antigen specific Thy $1.1+\mathrm{CD} 8+\mathrm{T}$ cells in the periphery at the indicated time points. Beginning seven days after treatment, all tumor areas were measured by multiplying the length of the longest dimension by the length of the perpendicular dimension. The mice were sacrificed once tumor size progressed past $200 \mathrm{~mm}^{2}$. The in vivo experiment was repeated two independent times and results were pooled.

\subsection{In vivo CD8+ $\mathrm{T}$ cell harvest and analysis}

To further probe the effect of treatment on CD8+ T cells, we used the same in vivo set up as used in Section 2.7. However, at day 11 post treatment, tumor size was measured, mice were sacrificed, and spleen and tumors were harvested and homogenized through a cell strainer. Tumor infiltrating lymphocytes (TILs) were isolated by a density separation technique, using Lympholyte-M (Cedarlane; Burlington, Ontario, Canada) according to the manufacturer's protocol. Splenocytes were treated with $4 \mathrm{~mL}$ of ACK lysis buffer for 1 minute to lyse red blood cells. Total immune cells were measured from the spleen and TILs with manual counting on a hemocytometer and a Trypan blue exclusion test to exclude dead cells. Immune cells were stained with surface staining antibodies anti-PD-1-PE/Cy7, antiThy1.1-APC, anti-CD8-APC/Cy7(Biolegend); Live/Dead-FITC (Life Technologies; Carlsbad, CA) for 30 minutes at $4{ }^{\circ} \mathrm{C}$. No more than 200,000 cells were used for any given condition. One sample per treatment group per organ was stained with anti-PD-1 mAb isotype antibody 
anti-RIgG2a-PE/Cy7. All samples were then washed and analyzed by flow cytometry on a BD FACSCalibur.

To look at the stimulatory potential of CD8+ T cells isolated from tumor-bearing animals of this in vivo experiment, we stimulated splenocytes isolated with micro-aAPC. Techniques previously described in 2.3. In Vitro Artificial Antigen Presenting Cell T-Cell Stimulation were followed with minor modifications. Briefly, micro Db-GP100/anti-CD28 mAb (37.51) aAPC on $4.5 \mu \mathrm{m}$ M-450 Epoxy Dynabeads (Life Technologies) were made at a 1:1 protein ratio, following manufacturer's protocol. Anti-CD28 monoclonal antibody was purchased from BioXCell (West Lebanon, NH). Splenocytes were stained with CFSE and DbGP100/anti-CD28 mAb micro aAPC were added to isolated splenocytes at a 1:1 ratio. Total dilution was assessed after three days of incubation by flow cytometry. Percent of diluted CD8+ T cells was measured by comparing flow cytometry histograms to non-stimulated controls for each condition studied.

\section{Results}

\subsection{PLGA-based aAPC synthesis and characterization}

PLGA-based aAPC were made as recently described [4]. Briefly, the core PLGA particle was synthesized by a single emulsion technique. The particles were subsequently functionalized by EDC/NHS chemistry with two proteins to mimic the surface of the natural APC which relays two critical signals to CD8+ T cells [4]. We used a soluble major histocompatibility complex (MHC) Class I-Ig fusion protein to mimic signal 1 in the aAPC/CD8 + T cell interaction, and an agonistic monoclonal anti-CD28 antibody to serve as a costimulatory molecule to mimic signal 2 [17]. In this way, the aAPC are designed to activate only a particular cognate CD8+ T cell that recognizes the specific MHC with loaded melanoma antigen peptide as signal 1 , which differs from common aAPC synthesis that have anti-CD3 antibody to the surface that could interact with T cells without antigen specificity. 
Initially we evaluated the physical and chemical properties of the PLGA-based aAPC by imaging lyophilized particles by scanning electron microscopy (SEM) to determine particle morphology and size (Figure 1a). SEM images revealed that the conjugated particles were spherical in nature. Image analysis determined an average particle size of $4.42 \mu \mathrm{m}$ with a standard deviation of $1.45 \mu \mathrm{m}$ (Figure $1 \mathrm{~b}$ ). We designed the aAPC to be approximately 4-5 $\mu \mathrm{m}$ in size as this is large enough to mimic the length scale of biological antigen presenting cells and also small enough to avoid pulmonary embolism following systemic injection.

We further looked to optimize protein conjugation and biodegradable particle stability by analyzing the surface protein content over various reaction times. To evaluate the surface protein content, we stained the particles with fluorescent monoclonal antibodies for the conjugated proteins, and measured the effect of incubation time of activated particle and protein on conjugation efficiency over a 48 hour period (Figure 1c). There is a clear increase in both pMHC and anti-CD28 mAb content at longer incubation times, maximally approximately $10 \%$ and $3 \%$ at 48 hours, respectively. This is an important finding for future manufacturing of biodegradable aAPCs; thus, we synthesized all subsequent particles for this study by incubating for 48 hours with protein. 
A.

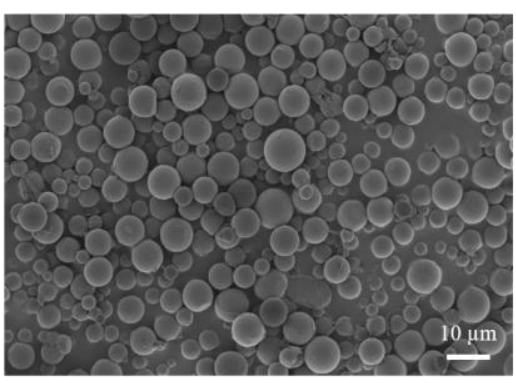

C.

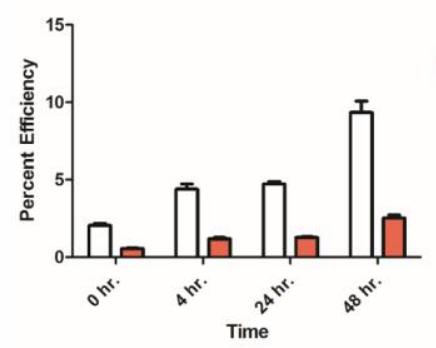

E.

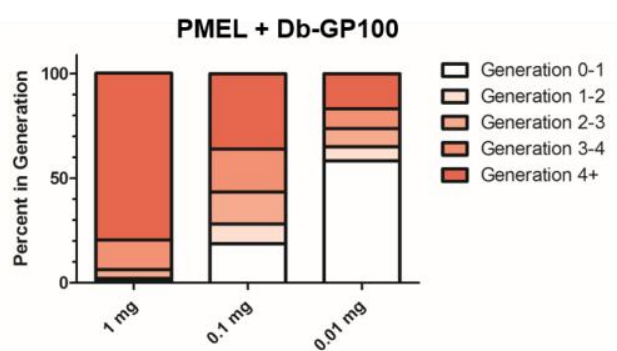

F..

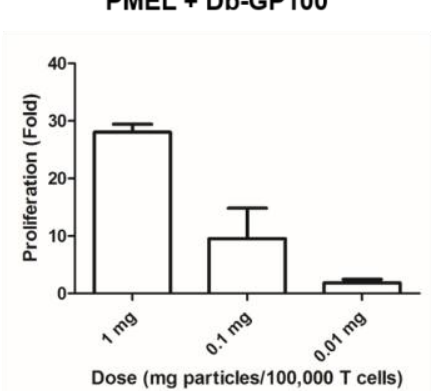

B.

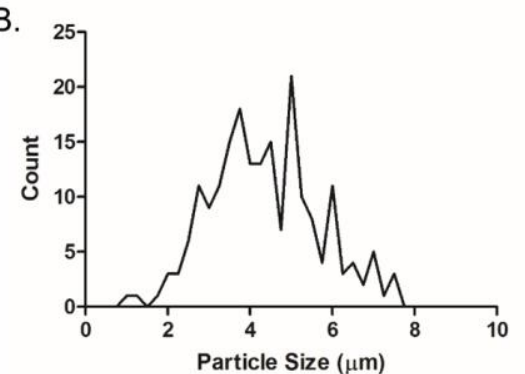

D.
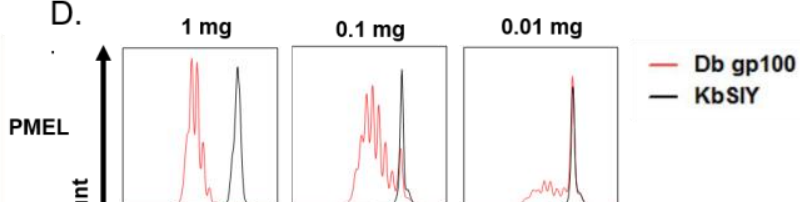

$2 \mathrm{C}$

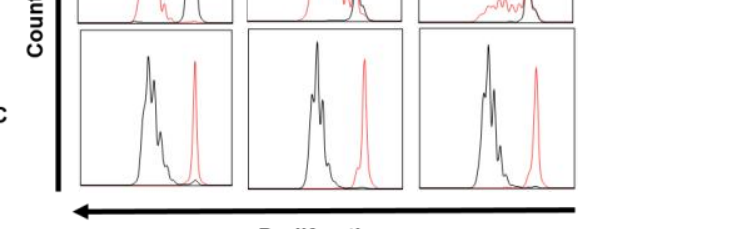

Proliferation

$2 \mathrm{C}+\mathrm{KbSIY}$

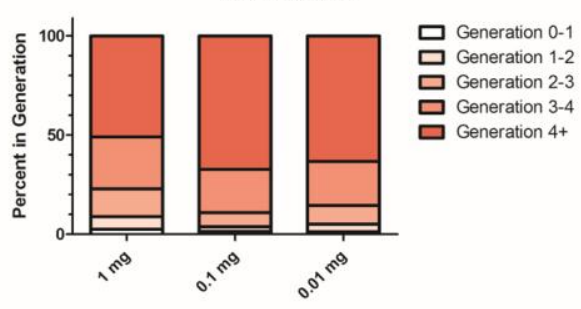

$2 \mathrm{C}+\mathrm{KbSIY}$

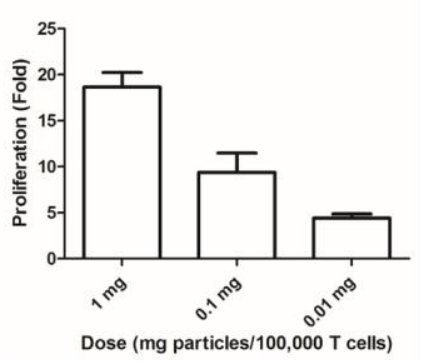

Figure 1. aAPC characterization and functional assessment. (a) SEM micrographs of conjugated aAPC microparticles. (b) Particle size distribution of conjugated aAPC microparticles as evaluated by image analysis of SEM micrographs. (c) Particle protein content on aAPC microparticles as evaluated by fluorescent antibody staining of the particles and measured by a fluorescent plate reader. Conjugation efficiency is defined in Materials and Methods. (d) Antigen specific CD8+ T cell stimulation capabilities of aAPC microparticles. CFSE dilution of CD8+ T cells from either PMEL or 2C TCR transgenic mice incubated for 3 days with indicated dose of particles $(1 \mathrm{mg}, 0.1 \mathrm{mg}, 0.01 \mathrm{mg})$ functionalized with the indicated antigen, GP100 or SIY peptide. (e) Generation analysis of CFSE dilution data in (d) indicates antigen specific proliferation of PMEL and 2C CD8+ T cells in response to stimulation by Db-GP100 (left) or Kb-SIY (right) aAPC microparticles. (f) Fold proliferation calculated by day 7 cell counts after stimulation with the Db-GP100 (left) or Kb-SIY (right) aAPC microparticles indicates antigen specific proliferation of CD8+ T cells. Error bars are SEM of $n=3$ replicates. 


\section{2. aAPC stimulate peptide-specific CD8+ T cells}

Biologically, one of the most important properties of an aAPC is the capability to specifically stimulate $\mathrm{T}$ cells of interest. This is important because non-specific activation would be problematic as it could lead to off-target inflammation and autoimmunity events. To that end we evaluated the ability of our aAPC to specifically stimulate CD8+ T cells from two primary transgenic mice (PMEL or $2 \mathrm{C}$ ) whose CD8+ T cells are all specific for the same peptide-MHC. The particles were functionalized with either Db-GP100 (cognate to PMEL $\mathrm{CD} 8+\mathrm{T}$ cells) or $\mathrm{Kb}-\mathrm{SIY}$ (cognate to $2 \mathrm{C} \mathrm{CD8}+\mathrm{T}$ cells) peptide-MHC and an anti-CD28 costimulatory monoclonal antibody. CD8+ T cell activation was quantified by two measurements of cell proliferation - CFSE dilution and day 7 cell counts.

Each particle type was separately incubated with CFSE-labeled 2C or PMEL CD8+ T cells, and CFSE dilution was analyzed by flow cytometry after three days. In this assay, if the CD8+ T cells are stimulated by the aAPC, they rapidly divide, diluting the CFSE dye between daughter cells following each division. Effective stimulation of CD8+ T cells occurred only in the case of a cognate antigen/CD8+ T cell match as seen by CFSE dilution (Figure 1d).

Besides antigen-specificity, we also studied the antigen-specific dosing of aAPC to help guide in vitro and in vivo studies with checkpoint therapy. Nearly all CD8+ T cells divided one or more times at the highest $1 \mathrm{mg}$ dose of aAPC. CD8+ T cell expansion was dose dependent as evidenced by CD8+ T cell generation analysis of CFSE data (Figure 1d,e). Nearly $80 \%$ of PMEL CD8+ T cells divided 4 or more times at a $1 \mathrm{mg}$ particle dose as compared to only $15 \%$ at a $0.01 \mathrm{mg}$ dose. For $2 \mathrm{C} \mathrm{CD} 8+\mathrm{T}$ cells, which have a lower activation threshold, robust proliferation was seen across all doses studied (Figure 1d,e).

Cell counts after 7 days of stimulation confirmed the dose-dependence of aAPC-based activation in both transgenic systems (Figure 1f). At the highest aAPC particle dose of $1 \mathrm{mg}$, PMEL CD8+ T cells reached approximately 30-fold expansion and 2C CD8+ T cells reached 
approximately 20 -fold expansion which is similar to our previously reported proliferation levels for spherical PLGA aAPCs [4].

\subsection{Anti-PD-1 mAb and aAPC activate cognate CD8+ $\mathrm{T}$ cells in vitro}

We hypothesized that simultaneous PD-1 blockade would further enhance activation of cognate cells for adoptive cell transfer. To investigate this, we first developed an in vitro system to mimic some of the major immunosuppressive characteristics of the tumor microenvironment. We utilized PD-L1 ${ }^{\text {hi }}$ target tumor cells and pre-activated CD8+ T cells that express PD-1 (schematic, Figure 2a).

To establish the in vitro system, $2 \mathrm{C} \mathrm{CD8}+\mathrm{T}$ cells were stimulated with cognate aAPC on days 0 and 4 to upregulate PD- 1 expression. This resulted in elevated PD- 1 expression by day 8 compared to naïve cells (Figure 2b). B16-SIY murine melanoma cells which express the cognate $\mathrm{Kb}-\mathrm{SIY}$ pMHC, and noncognate B16-F10 murine melanoma cells, were treated with IFN- $\gamma$ for 48 hours to upregulate PD-L1 expression (Figure 2c). All IFN- $\gamma$ was removed from B16-SIY cells from prior treatment, as confirmed by undetectable levels of the cytokine from B16-SIY cells alone (Figure 2d). 


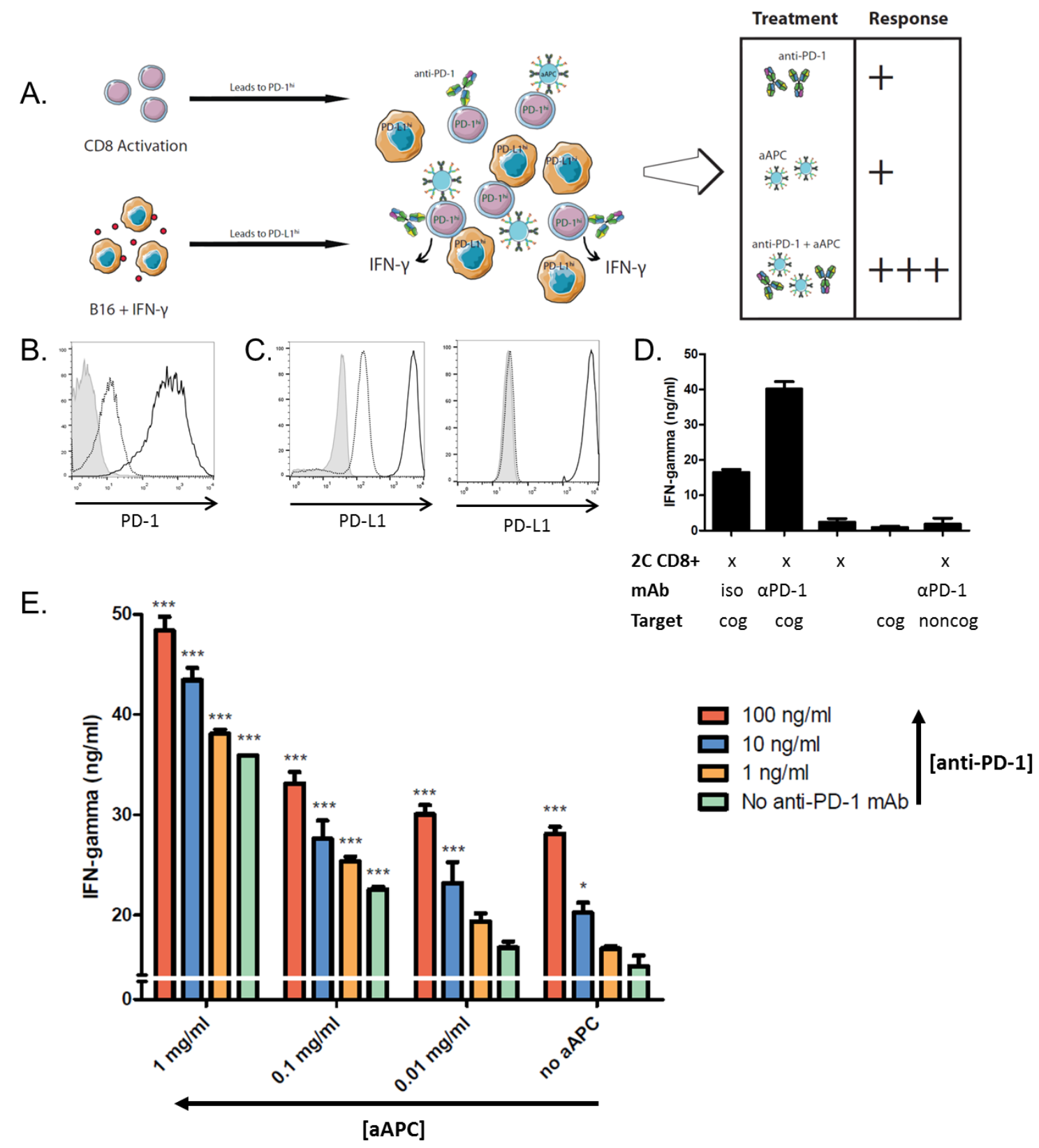

Figure 2. aAPC and anti-PD-1 mAb show greater CD8+ T cell activation in combination. (a) Schematic of in vitro tumor microenvironment model system. 2C CD8+ T cells were isolated and stimulated with a 1:1 cell:aAPC ratio and B16-SIY and B16-F10 cells were incubated with $20 \mathrm{ng} / \mathrm{ml}$ IFN- $\gamma$. Activated 2C CD8+ T cells and B16 cells were purified from aAPC and IFN- $\gamma$, respectively, and combined at a 1:1 effector to target ratio with additional aAPC in the presence or absence of anti-PD-1 mAb. IFN- $\gamma$ release was measured by ELISA after 18 hours. (b) CD8+ T cells are PD- $1^{\text {hi }}$ after dual aAPC activation (black), compared to expression on naive cells (dotted) and isotype (grey). (c) B16-SIY (left) and B16-F10 (right) are PD-L1 ${ }^{\text {hi }}$ after IFN- $\gamma$ treatment (black) as compared to untreated cells (dotted) and isotype (grey). (d) PD- $1^{\text {hi }} 2 \mathrm{C} \mathrm{CD} 8+$ T cells and target PD-L1 ${ }^{\text {hi }}$ B16-SIY (cognate; cog) or B16-F10 (noncognate; noncog) were co-incubated at a 1:1 ratio in the presence of $10 \mathrm{ug} / \mathrm{ml}$ anti-PD1 $\mathrm{mAb}$ or isotype control (iso). Anti-PD-1 mAb increased CD8+ T cell IFN- $\gamma$ release as compared to isotype. No IFN- $\gamma$ release was measured in response to CD8+ T cells and noncognate B16-F10 cells incubated with anti-PD-1 mAb. Significance was measured by one-way ANOVA with Dunnett's post-test. (e) CD8+ T cells secrete more IFN- $\gamma$ in response to increasing doses of 
both aAPC and anti-PD-1 mAb. Error bars represent SEM, and significance compared to no treatment (i.e. no aAPC, anti-PD-1) is shown $(*=\mathrm{p}<0.05, * *=\mathrm{p}<0.01$, *** $=\mathrm{p}<0.001)$. Both aAPC and anti-PD-1 significantly affect IFN $-\gamma\left(\mathrm{p}<0.001 ; \mathrm{F}_{3,40}=226.4\right.$ and $\mathrm{F}_{3,40}=88.32$, respectively) by two-way ANOVA.

To validate that the resultant cell phenotypes can model the effect of PD-1 blockade in vitro, we first assessed IFN- $\gamma$ release from CD8+ T cells in response to anti-PD-1 mAb alone. 8-day activated PD- $1^{\text {hi }} 2 \mathrm{C} \mathrm{CD8}+\mathrm{T}$ cells and 48 hour treated PD-L1 ${ }^{\text {hi }}$ B16-SIY cells were coincubated at a 1:1 ratio in the presence or absence of $10 \mu \mathrm{g} / \mathrm{ml}$ anti-PD- $1 \mathrm{mAb}$ or isotype control antibody. IFN- $\gamma$ secretion, a marker of T cell activation, was 2.5 -fold higher in the presence of anti-PD-1 mAb (Figure 2d). Importantly, anti-PD-1 mAb did not stimulate CD8+ T cells in the absence of a cognate signal 1, indicated by co-incubation with noncognate B16F10 cells. These results demonstrate that this model can assess synergy between anti-PD-1 $\mathrm{mAb}$ and aAPC-based CD8+ $\mathrm{T}$ cell activation in vitro.

Next, we sought to investigate the combinatorial power of aAPC and anti-PD-1 mAb treatment in vitro. We hypothesized that this two-hit approach would disrupt the inhibitory PD-1/PD-L1 pathway which is upregulated during stimulation and therefore lead to greater CD8+ T cell activation by aAPC. $2 \mathrm{C} \mathrm{CD} 8+\mathrm{T}$ cells were stimulated for five days with cognate aAPC and B16-SIY cells were primed with IFN- $\gamma$ as previously described. CD8+ T cells and B16 cells were co-incubated at a 1:1 ratio in the presence of titrating amounts of cognate PLGA aAPC and anti-PD-1 mAb alone or in combination, and response was quantified by IFN- $\gamma$ release (schematic, Figure $2 a$ ). While CD8+ T cells secreted minimal levels of IFN- $\gamma$ with no aAPC or anti-PD-1 mAb treatment, and either treatment alone increased activation in a dose-responsive manner (Figure 2e), the combination of aAPC and anti-PD-1 mAb resulted in the greatest activation - over 3.5-fold more IFN- $\gamma$ secretion over no treatment. No further increase in IFN- $\gamma$ release was seen with higher anti-PD-1 mAb doses. 
A.

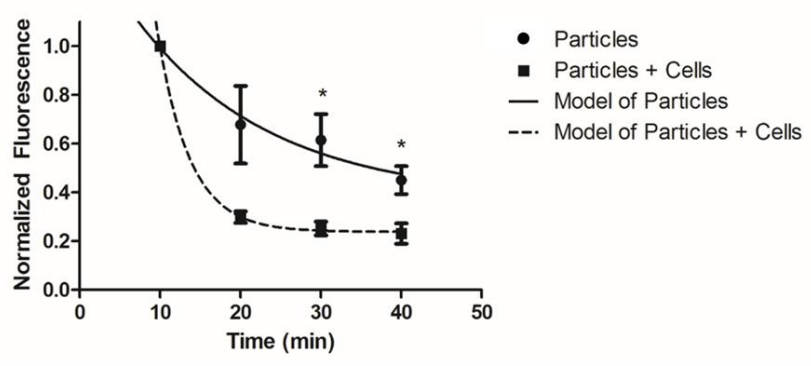

B.

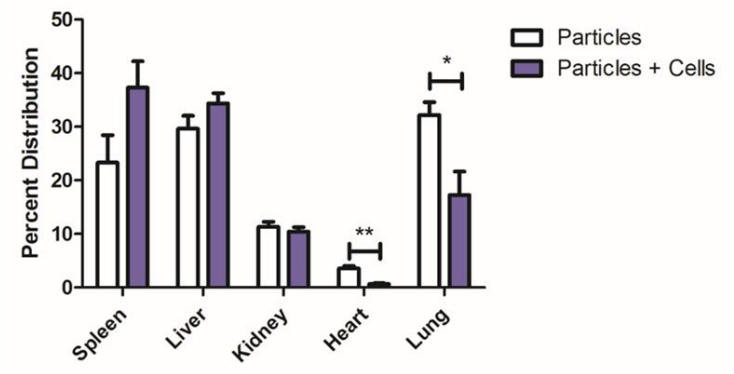

Figure 3. Co-administration of aAPC with CD8+ T cells impacts aAPC biodistribution. (a) Blood was collected retroorbitally following intravenous administration of IR fluorescence labeled particles alone or incubated with cells. Blood was imaged and fluorescence was quantified and normalized to the first time point collected. Asterisk indicates time points at which normalized data was significantly different between the two groups. Lines through the points denote first order exponential decay curves fit to the data. In the presence of cells, the aAPC particles are eliminated faster than without cells. (b) At 24 hrs post-aAPC administration, the organs were dissected out, imaged, and quantified for fluorescence. In the presence of cells, the aAPC particles resist getting trapped in the lungs $(\mathrm{p}<0.05)$ or the heart $(\mathrm{p}<0.01)$, and trend towards accumulating more in the spleen $(\mathrm{p}=0.1)$. Error bars are SEM of $\mathrm{n}=3$ replicates. $(*=\mathrm{p}<0.05, * *=\mathrm{p}<0.01)$

\subsection{PLGA aAPC biodistribution}

We next sought to demonstrate the biodistribution of aAPC with and without adoptively transferred CD8+ T cells. PLGA aAPC particles encapsulating a near infrared dye were injected intravenously into C57BL/6 mice. Particles were injected either alone or after a co-incubation with cognate CD8+ T cells to assess their impact on particle distribution. We serially collected blood and imaged the samples to track fluorescence of the particles (Figure 3a). There was a noticeably faster elimination of the particles in the presence of cells compared to particles alone. A single exponential decay curve was fit to each data set, and it was determined that the effective half-life of the aAPC alone was 11.6 min and aAPC in the presence of cells was $2.8 \mathrm{~min}$. Thus, by $40 \mathrm{~min}$, multiple half-lives occurred, sufficient for the majority of the particles to clear the blood and sufficient to calculate the respective halflives. At 30 and 40 minutes post injection, there were significantly more aAPC particles still in circulation when particles were injected alone as compared to particles co-injected with cells (Figure 3a). The particles were not designed to have long circulation in the blood and 
we anticipated that they would be readily cleared within $40 \mathrm{~min}$ from the blood to these organs of interest.

24 hours post administration, the animals were sacrificed and the spleen, liver, kidney, heart, and lung were harvested for fluorescence quantification (Figure 3b). In both the aAPC alone and the aAPC with cells groups the major organs for accumulation were the spleen and the liver. The aAPC in the presence of cells potentially had $60.1 \%$ greater accumulation in the spleen than the aAPC alone. With regard to the lung $(\mathrm{p}<0.05)$ and heart $(\mathrm{p}<0.01)$ it was concluded that there were fewer aAPC particles trapped in these tissues when the aAPC were in the presence of cells compared to aAPC alone. Thus, it was concluded that aAPC and CD8+ T cells should be co-injected for optimal localization to lymphoid organs.

This biodistribution study also demonstrated that systemic injection of the aAPC particles with or without cells did not cause an acute safety risk such as embolism. Systemic injection of these micron-scale biomimetic aAPC is a new administration route as we have previously used similar aAPC particles only sub-cutaneously [4]. We found the intravenous injections to be well tolerated in all animals. This approach used for the biodistribution studies also highlights another potential advantage of these particular PLGA aAPC biomaterials as it validates that they are capable of co-encapsulating imaging or other agents internally, while orthogonally allowing presentation of biomolecules from their surfaces.

Our studies also suggest a trend that the aAPCs + CD8 + T cells resisted the first pass off-target clearance tissue (lung) to accumulate more at the on target immune tissue (spleen). This observed biodistribution to the spleen may help explain the therapeutic outcome. By investigating the interaction and biodistribution of the aAPC biomaterial in vivo for the first time, we observed that co-administration with $\mathrm{T}$ cells does make a difference. This is a novel approach to T cell immunotherapy as T cells are commonly activated ex vivo and then separated from their agonists prior to in vivo administration. 


\subsection{Anti-PD-1 mAb synergizes with biodegradable aAPC in vivo to delay tumor growth and extend survival}

To evaluate the therapeutic potential of combination biodegradable aAPC and antiPD-1 mAb therapies, we assessed their efficacy alone or in combination in an adoptive immunotherapy melanoma tumor treatment model. Thy1.2+ C5BL/6 mice were inoculated subcutaneously in the right flank with B16-F10 murine melanoma cells (schematic, Figure 4a). Three days later, all mice were irradiated with a sub-lethal dose of radiation to induce transient lymphopenia. On the following day, naïve cognate Thy1.1+ PMEL CD8+ T cells alone or co-incubated with PLGA aAPC were injected intravenously into treated mice. AntiPD-1 mAb was given intraperitoneally on the same day and the day after aAPC and CD8+ T cell treatment, as per standard anti-PD-1 mAb treatment [33]. Likewise, PD-1 expression was shown to be upregulated by the CD8+ T cells within 7-24 hours of aAPC activation (Supplemental Figure 1), and early anti-PD-1 mAb treatment ensured an effect on the stimulated cells. Peripheral blood samples were taken from the mice to evaluate antigen specific CD8+ T cell proliferation of the adoptively transferred cells, and tumor size was measured every other day (see Materials and Methods, 2.7).

Analysis of the circulating CD8+ T cell population revealed a proliferative advantage of the adoptively transferred cells in the dual treatment group (Figure 4b). Significantly higher percentages of Thy1.1+ antigen specific CD8+ T cells were seen in the periphery of mice treated with the combined aAPC and anti-PD-1 mAb treatment as opposed to aAPC or antiPD-1 mAb alone ( $\mathrm{p}<0.001$ on days 10 and 13). There was on average a 3-4 fold increase in proliferation of $\mathrm{CD} 8+\mathrm{T}$ cells in the combination treatment group over the anti-PD-1 mAb alone group and a 2 fold proliferative advantage of the combination treatment group over the aAPC alone group. 
A therapeutic benefit of the combination treatment was also seen as determined by inhibition of tumor growth and prolonged survival (Figure 4c-e). There was a statistically significant reduction in tumor burden for the combination treatment group compared to all other groups past day 15 (Figure 4c). No single treatment significantly delayed tumor growth. By day 15 , the combination treatment group had an average tumor size of $140 \mathrm{~mm}^{2}$ - over a $30 \%$ reduction in tumor size from the no treatment and single treatment groups with all $\mathrm{p}$ values being less than 0.05 . Additionally, the survival of the animals was extended only in the combination treatment group $(\mathrm{p}<0.05)($ Figure $4 \mathrm{~d})$. No mice in the non-treated and single treatment groups survived past day 20 , whereas $45 \%$ of mice in the dual treatment group survived past this time point. This survival advantage was statistically significant compared to all other groups. 


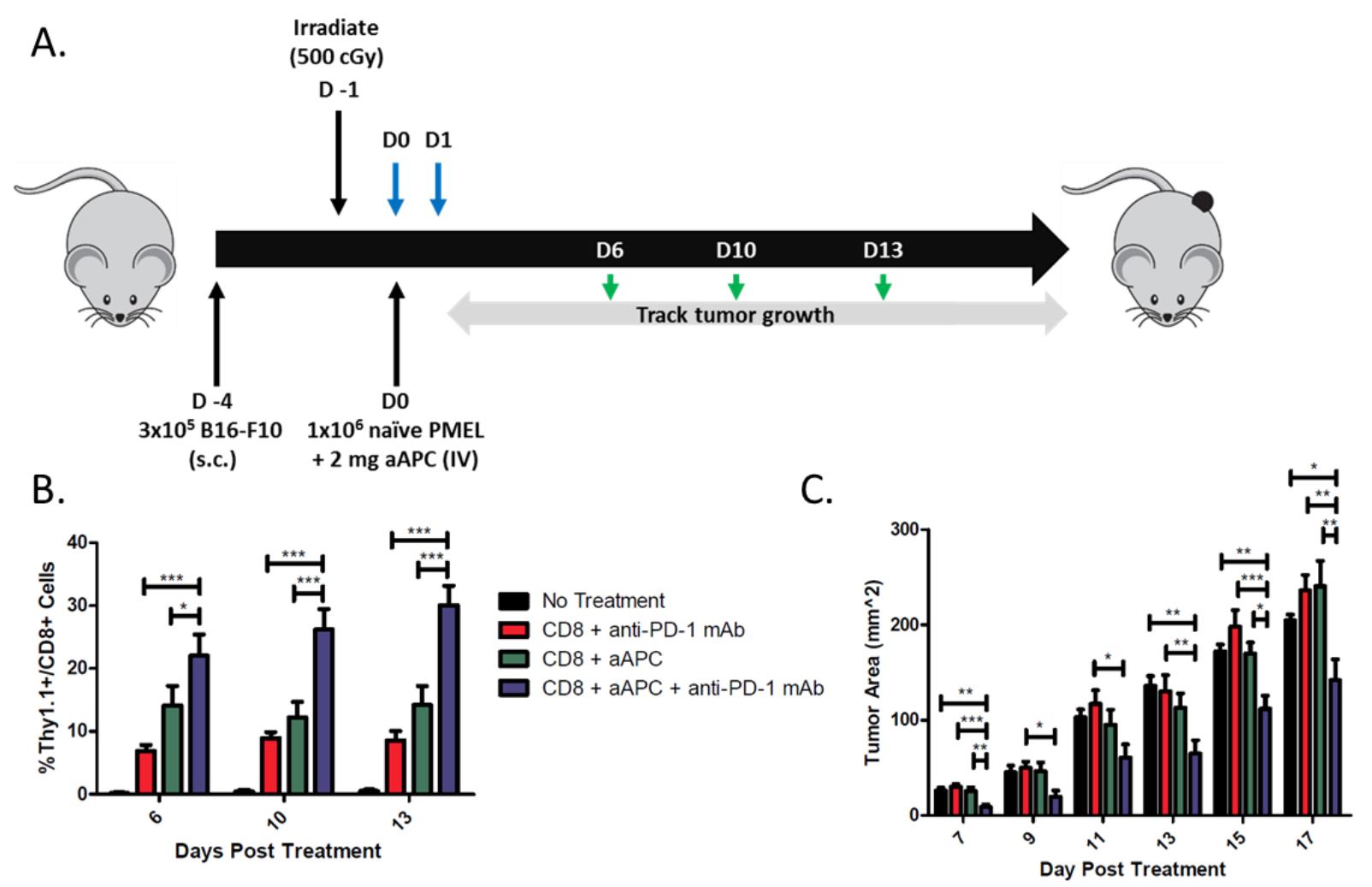

D.

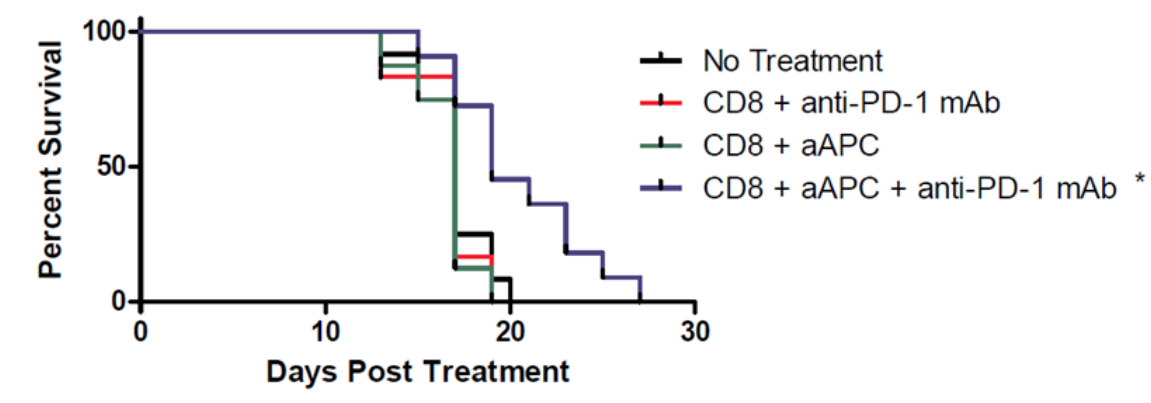

Figure 4. Anti-PD1 $\mathrm{mAb}$ and aAPC synergize to mediate anti-tumor activity in vivo. (a) Schematic of the adoptive transfer experiment. Blue arrows indicate anti-PD-1 mAb treatment, green arrows indicate blood sampling days. (b) Percent of CD8+ T cells in peripheral blood that are Thy1.1+. Anti-PD1 mAb and aAPC dual therapy mediated the best proliferation of antigen-specific CD8+ T cells in vivo. Significance evaluated with one-way ANOVA with Tukey's post-test. All treatment groups were significantly different from no treatment at all time points. (c) Tumor measurements indicate an anti-tumor response was mediated by the dual therapy group. Significance measured by one-way ANOVA with Tukey's post-test. (d) Kaplan-Meier survival plots indicate that dual therapy mediates the best survival. Only combination treatment resulted in significantly extended survival compared to no treatment by Log-rank test $(\mathrm{p}<0.05)$. Error bars represent the standard error of $n=8-12$ mice/group. Results from two independent experiments are pooled. $(* \mathrm{p}<0.05, * * \mathrm{p}<0.01, * * * \mathrm{p}<0.001)$ 


\subsection{Dual anti-PD-1 mAb and aAPC treatment results in superior tumor-specific CD8+}

\section{T cells in vivo}

To understand mechanistically how the combination of aAPC and anti-PD-1 mAb treatment affects tumor-specific CD8+ T cells in vivo, we analyzed phenotypic and functional changes in adoptively transferred CD8+ T cells in the various treatment groups. Thy $1.2+$ C5BL/6 mice were inoculated subcutaneously in the right flank with B16-F10 murine melanoma cells and sublethally irradiated after three days. Mice received either Thy $1.1+$ PMEL CD8+ T cells alone or co-incubated with aAPC, and were treated with anti-PD-1 mAb as described above. Eleven days after treatment, tumor infiltrating lymphocytes (TILs) and splenocytes were harvested and adoptively transferred cells were identified by the presence of Thy1.1+ on the cell surface (see Materials and Methods, 2.8).

Thy 1.1+ CD8+ adoptively transferred PMEL T cells in the TILs expressed significantly higher levels of the immunosuppressive PD-1 molecule in the absence of antiPD-1 treatment (Figure 5a). A similar trend was evident in the spleen, where adoptively transferred cells expressed higher PD-1 levels in the absence of any additional treatment (Figure 5b). PD-1 expression was decreased to a greater extent within TILs as compared to the spleen, approximately 2-fold and 6-fold, respectively, likely because these cells are more immunosuppressed and thus express higher levels of PD-1 in the absence of treatment. Additionally, there was about half the percentage of Thy $1.1+\mathrm{CD} 8+\mathrm{T}$ cells in the spleen of mice lacking aAPC treatment as compared to dual treated mice (Figure 5c).

To investigate the functional capacity of the tumor-specific cells, we studied their ability to expand in response to re-stimulation. Isolated splenocytes from each mouse were stained with a CFSE dye and re-stimulated in vitro with aAPC expressing cognate Db-GP100 pMHC and anti-CD28 mAb to expand only tumor-specific cells. After three days, CFSE dilution was assessed by flow cytometry and expanded cells identified. CD8+ T cells from dual treated mice expanded about 2-3 fold more in response to antigen-specific re-stimulation 
than compared to non-treated and anti-PD-1 mAb only treated mice (Figure 5d). Together, this data shows that dual aAPC and anti-PD-1 mAb treatment leads to a change in expression of checkpoint molecules and increases the proliferative capacity of CD8+ T cells both within the tumor microenvironment and secondary lymphoid tissue. PD-1 blockade reduces CD8+ T cell PD-1 expression and aAPC treatment increases tumor-specific CD8+ T cell expansion and re-activation potential. Thus, combination therapy leads to a superior CD8+ $\mathrm{T}$ cell population by facilitating both lower expression of the immunosuppressive PD-1 molecule and increased proliferative and functional capacity. 
A
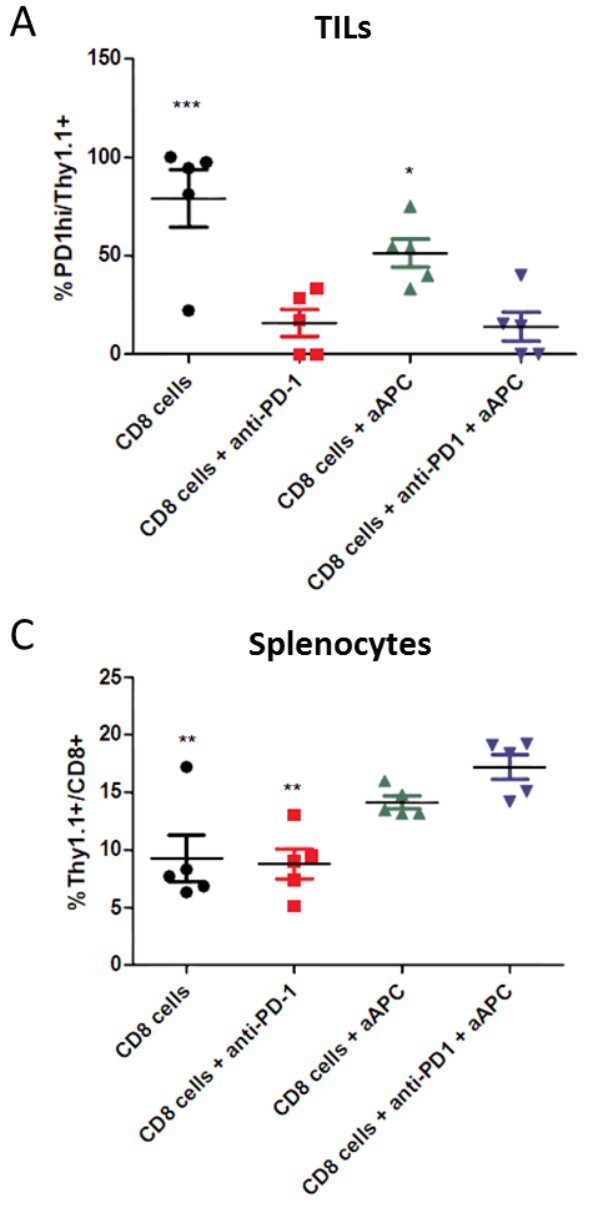

B

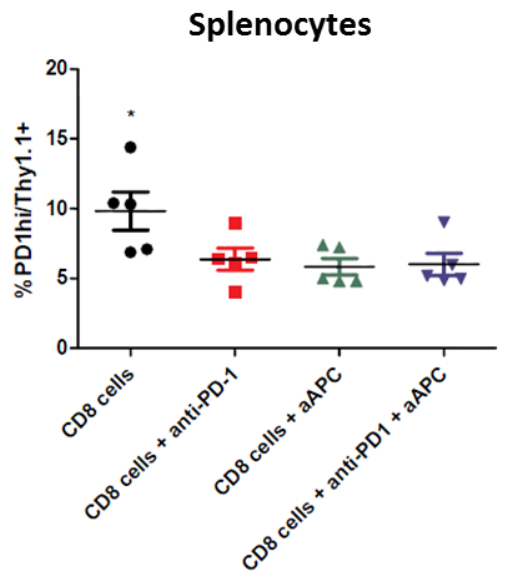

D

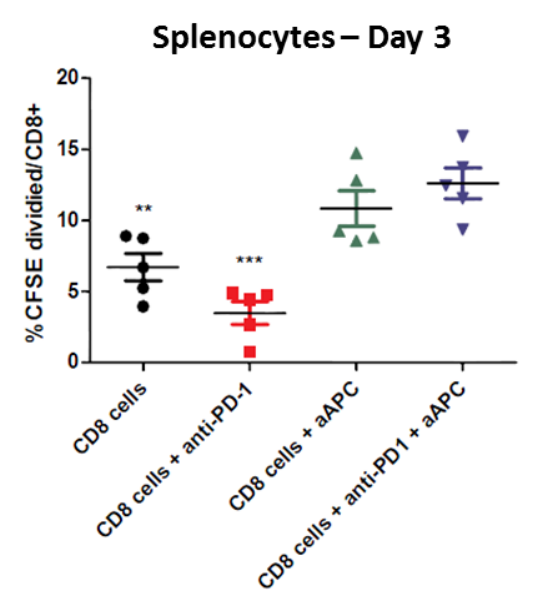

Figure 5. Anti-PD-1 mAb and aAPC combination therapy decreases PD-1 expression and increases expansion of tumor-specific CD8+ T cells. C57BL/6 mice were inoculated with B16-F10 tumors on day -4 and sublethally irradiated on day -1 . On day 0 , mice received an IV injection of PMEL Thy 1.1+ CD8+ T cells either alone or co-incubated with cognate aAPC. Two of the groups also received an IP injection of anti-PD-1 mAb on days 0 and 1.

Splenocytes and TILs were harvested on day 11. (a) Thy1.1+ TILs of mice not treated with anti-PD-1 mAb had significantly elevated PD-1 expression as compared to dual treated mice. (b) Thy $1.1+$ cells in the spleen of non-treated mice also had significantly elevated PD-1 expression as compared to dual treatment. (c) CD8+ T cells within the spleen of mice not treated with aAPC had a significantly lower percentage of Thy1.1+ tumor-specific cells as compared to dual treatment. (d) Splenocytes were CFSE stained and re-stimulated with DbGP100/anti-CD28 mAb aAPC, and CFSE dilution was assessed after 3 days. There was significantly less expansion of tumor-specific CD8+ T cells in mice not treated with aAPC. Significance measured by one-way ANOVA with Dunnett's post-test comparing all groups to dual treatment $(* \mathrm{p}<0.05, * * \mathrm{p}<0.01, * * * \mathrm{p}<0.001)$.

\section{Discussion}

Stimulating a tumor-specific cytotoxic CD8+ T cell response is a promising approach in cancer immunotherapy, although several hurdles still exist in generating a population of cells that is both optimally effective and persistent. Often, very large numbers of activated T 
cells, up to $10^{11}$ [12], are necessary for an objective response. Advancements in biomimetic and biodegradable aAPC have made robust activation for clinical therapy possible, although strong activation upregulates immunosuppressive surface molecules on the CD8+ T cells such as PD-1. As progress is made in the field of biomaterials for robust $\mathrm{T}$ cell activation, it is likewise necessary to understand their interaction with other conventional immunotherapeutics, including new clinical standards of care, to counterbalance the resultant inhibitory phenotype.

Here, we have characterized the synergistic interaction between biodegradable PLGA aAPC and PD-1 checkpoint blockade for murine tumor control in an adoptive transfer model. PLGA-based aAPC were chosen as they are effective T cell stimulators, and, due to wellknown experience with PLGA particles in the body, offer translational potential for tumorspecific CD8+ T cell expansion in vivo. We showed antigen-specific stimulation of CD8+ T cells in response to PLGA aAPC conjugated with two different peptide-MHC complexes. The aAPC are able to induce robust expansion in each of the tested cell types.

However, the immune system has developed ways to self-regulate itself by upregulating immunosuppressive molecules in response to activation. In this nature, activated CD8+ T cells express high levels of PD-1 which is important biologically to control autoimmunity, but detrimental in cancer immunotherapy where a strong anti-tumor response is desired. Tumors take advantage of this biological process and express the ligand for PD-1, PD-L1, to inhibit an anti-tumor CD8+ T cell response. Our in vitro model of PD- ${ }^{\text {hi }}$ CD8+ T cells and PD-L1 ${ }^{\text {hi }}$ tumor cells, mimicking those that would be found within the tumor microenvironment of a patient, showed increased CD8+ T cell activation in response to the combination of aAPC stimulation and PD-1 blockade. While both therapeutics alone increased activation, the combination was able to stimulate CD8+ T cells while turning off their "rheostat" and led to a further enhanced antigen-specific response. 
Finally, we sought to study the effectiveness of combination treatment in vivo since the dynamics of the tumor microenvironment can play a significant role in the response. The aAPC were first demonstrated to circulate systemically, both with and without a co-injection of cognate CD8+ T cells. When aAPC were co-injected with CD8+ T cells, they spent less time in circulation and accumulated less in off-target sites such as the lung and heart. This comparison is both interesting and important, as the intrinsic honing capabilities of CD8+ T cells may cause accumulation of aAPC in immune-dense sites. Indeed, we did see a trend towards higher aAPC accumulation in secondary lymphoid tissue - the spleen. While this $>50 \%$ change in distribution was not significant under the conditions studied, it may help to explain the statistically shorter circulation half-life of particles co-injected with cells along with the greater accumulation of tumor-specific CD8 $+\mathrm{T}$ cells in the spleens of mice treated with particles and cells in vivo.

Combination of aAPC expressing tumor antigens and anti-PD-1 mAb treatment resulted in delayed tumor growth and extended survival in vivo in an adoptive transfer model of naïve tumor specific cells. While many adoptive immunotherapy approaches begin with pre-activated CD8+ T cells, our approach demonstrates significant anti-tumor activity in the absence of prior stimulation and thus reduces the time, cost, and technical hurdles associated with ex vivo cell culture. Importantly, this is the first time this PLGA aAPC-based therapeutic was effective in a therapeutic rather than prophylactic tumor model as well as the first time that it has been evaluated following systemic administration [4]. The therapeutic effect was shown to be due to increased proliferation of the adoptively transferred tumor-specific cells both in the peripheral blood and spleen in response to aAPC transfer, as well as decreased PD1 expression by these cells in the spleen and tumor in response to anti-PD- $1 \mathrm{mAb}$ treatment. Despite only two anti-PD-1 mAb treatments on days 0 and 1, there was still a significant nearly 6-fold decrease in CD8+ T cell PD-1 expression 10 days after the last anti-PD-1 administration. Importantly, this strong stimulation of the tumor-specific CD8+ T cells did not 
lead to unresponsiveness - CD8+ T cells from the spleen of the dual treatment group also expanded the greatest amount upon re-stimulation ex vivo.

In summary, anti-PD-1 immunotherapy unleashes more of the aAPC-activated CD8+ T cells to perform effector function whereas without anti-PD-1 mAb therapy more aAPCactivated CD8+ T cells were ineffective due to the immunosuppressive tumor cells expressing PD-L1. Furthermore, aAPC activate and increase the number of CD8+ T cells that anti-PD1 therapy can target and affect. CD8+ T cells not activated by aAPC do not proliferate as much or upregulate PD1, thus decreasing the total effect of the checkpoint blockade immunotherapy. Therefore, both therapies complement and synergize to provide a more potent tumor immunotherapy.

\section{Conclusion}

Here we have developed a biomaterial-based combinatorial cancer immunotherapy. This therapeutic simultaneously activates cytotoxic CD8+ T cells while reducing the immune dampening effects of the tumor microenvironment. We have developed a biomimetic PLGAbased aAPC that can, in an antigen specific fashion, stimulate cancer-targeting CD8+ T cells and synergize with PD-1 checkpoint blockade. The aAPC particles generate a cytotoxic response against melanoma cells in vitro and, for the first time, these aAPC have been shown effective in an in vivo tumor treatment model following a single intravenous injection. The combined therapy was able to mediate a reduction in tumor burden and increase median survival time. As the aAPC are composed of PLGA, a biodegradable material that has a trackrecord of safe use in the clinic with related technologies, these advanced immunostimulatory materials may be promising for combination treatment in the clinical application of cancer immunotherapy. 


\section{Acknowledgements}

The authors thank the JHU-Coulter Translational Partnership, the TEDCO Maryland Innovation Initiative, The Troper Wojcicki Foundation, the NIH (R01-EB016721, P30EY001865, AI44129, and CA108835), and sponsored research agreements with Miltenyi Biotec and NexImmune for support of this research. Antibody and lymphocyte images reproduced under a Creative Commons License from Servier Medical Art (http://www.servier.com/Powerpoint-image-bank). RAM thanks the NIH Cancer Nanotechnology Training Center (R25CA153952) at the JHU Institute for Nanobiotechnology and the Achievement Rewards for College Scientists (ARCS) for fellowship support. AKK thanks the National Science Foundation (DGE-1232825), the NIH Cancer Nanotechnology Training Center at the JHU Institute for Nanobiotechnology (2T32CA153952-06), and the National Cancer Institute of the NIH (F31CA206344) for fellowship support. JWH is supported by a National Science Foundation Graduate Research Fellowship (DGE-1232825). 
[1] Purwada A, Roy K, Singh A. Engineering vaccines and niches for immune modulation. Acta Biomater. 2014;10(4):1728-1740.

[2] Danhier F, Ansorena E, Silva JM, Coco R, Le Breton A, Préat V. PLGA-based nanoparticles: An overview of biomedical applications. J Control Release. 2012;161(2):505-522.

[3] Jhunjhunwala S, Raimondi G, Glowacki AJ, Hall SJ, Maskarinec D, Thorne SH, Thomson AW, Little SR. Bioinspired controlled release of CCL22 recruits regulatory T cells in vivo. Adv Mater. 2012;24(35):4735-4738.

[4] Sunshine JC, Perica K, Schneck JP, Green JJ. Particle shape dependence of CD8+ T cell activation by artificial antigen presenting cells. Biomaterials. 2014;35(1):269-77.

[5] Oelke M, Schneck JP. Overview of a HLA-Ig based "Lego-like system" for T cell monitoring, modulation and expansion. Immunol Res. 2010;47(1-3):248-56.

[6] Durai M, Krueger C, Ye Z, Cheng L, Mackensen A, Oelke M, Schneck JP. In vivo functional efficacy of tumor-specific T cells expanded using HLA-Ig based artificial antigen presenting cells (aAPC). Cancer Immunol Immunother. 2009;58(2):209-20.

[7] Sasawatari S, Tadaki T, Isogai M, Takahara M, Nieda M, Kakimi K. Efficient priming and expansion of antigen-specific CD8+ T cells by a novel cell-based artificial APC. Immunol Cell Biol. 2006;84(6):512-21.

[8] Latouche JB, Sadelain M. Induction of human cytotoxic T lymphocytes by artificial antigen-presenting cells. Nat Biotechnol. 2000;18(4):405-9.

[9] Ye Q, Loisiou M, Levine BL, Suhoski MM, Riley JL, June CH, Coukos G, Powell DJ Jr. Engineered artificial antigen presenting cells facilitate direct and efficient expansion of tumor infiltrating lymphocytes. J Transl Med. 2011;9(1):131.

[10] Gong W, Ji M, Cao Z, Wang L, Qian Y, Hu M, Qian L, Pan X. Establishment and characterization of a cell based artificial antigen-presenting cell for expansion and activation of CD8+ T cells ex vivo. Cell Mol Immunol. 2008;5(1):47-53. 
[11] Maus M V, Thomas AK, Leonard DGB, Allman D, Addya K, Schlienger K, Riley JL, June $\mathrm{CH}$. Ex vivo expansion of polyclonal and antigen-specific cytotoxic T lymphocytes by artificial APCs expressing ligands for the T-cell receptor, CD28 and 41BB. Nat Biotechnol. 2002;20(2):143-8.

[12] Rosenberg SA, Restifo NP. Adoptive cell transfer as personalized immunotherapy for human cancer. Science. 2015;348(6230):62-68.

[13] Butler MO, Lee J-S, Ansén S, Neuberg D, Hodi FS, Murray AP, Drury L, Berezovskaya A, Mulligan RC, Nadler LM, Hirano N. Long-lived antitumor CD8+ lymphocytes for adoptive therapy generated using an artificial antigen-presenting cell. Clin Cancer Res. 2007;13(6):1857-67.

[14] Ugel S, Zoso A, De Santo C, Li Y, Marigo I, Zanovello P, Scarselli E, Cipriani B, Oelke M, Schneck JP, Brote V. In vivo administration of artificial antigen-presenting cells activates low-avidity T cells for treatment of cancer. Cancer Res. 2009;69(24):9376-84.

[15] Shen C, Cheng K, Miao S, Wang W, He Y, Meng F, Zhang J. Latex bead-based artificial antigen-presenting cells induce tumor-specific CTL responses in the native Tcell repertoires and inhibit tumor growth. Immunol Lett. 2013;150(1-2):1-11.

[16] Sunshine JC, Green JJ. Nanoengineering approaches to the design of artificial antigenpresenting cells. Nanomedicine. 2013;8(7):1173-89.

[17] Meyer R a, Sunshine JC, Perica K, Kosmides AK, Aje K, Schneck JP, Green JJ. Biodegradable Nanoellipsoidal Artifi cial Antigen Presenting Cells for Antigen Specific T-Cell Activation. Small. 2014:1-7.

[18] Steenblock ER, Fadel T, Labowsky M, Pober JS, Fahmy TM. An artificial antigenpresenting cell with paracrine delivery of IL-2 impacts the magnitude and direction of the T cell response. J Biol Chem. 2011;286(40):34883-92. 
[19] Steenblock ER, Fahmy TM. A comprehensive platform for ex vivo T-cell expansion based on biodegradable polymeric artificial antigen-presenting cells. Mol Ther. 2008; 16(4):765-72.

[20] Okazaki T, Chikuma S, Iwai Y, Fagarasan S, Honjo T. A rheostat for immune responses: the unique properties of PD-1 and their advantages for clinical application. Nat Immunol. 2013;14(12):1212-1218.

[21] Iwai Y, Ishida M, Tanaka Y, Okazaki T, Honjo T, Minato N. Involvement of PD-L1 on tumor cells in the escape from host immune system and tumor immunotherapy by PDL1 blockade. PNAS. 2002;99(19):12293-7.

[22] Pilon-Thomas S, Mackay A, Vohra N, Mulé JJ. Blockade of programmed death ligand 1 enhances the therapeutic efficacy of combination immunotherapy against melanoma. J Immunol. 2010;184(7):3442-9.

[23] Callahan MK, Wolchok JD. At the bedside: CTLA-4- and PD-1-blocking antibodies in cancer immunotherapy. J Leukoc Biol. 2013;94(1):1-13.

[24] Ascierto P a., Marincola FM. 2015: The Year of Anti-PD-1/PD-L1s Against Melanoma and Beyond. EBioMedicine. 2015;2(2):92-93.

[25] Robert C, Long G V., Brady B, et al. Nivolumab in previously untreated melanoma without BRAF mutation. N Engl J Med. 2015;372(4):320-30.

[26] Robert C, Ribas A, Wolchok JD, et al. Anti-programmed-death-receptor-1 treatment with pembrolizumab in ipilimumab-refractory advanced melanoma: a randomised dose-comparison cohort of a phase 1 trial. Lancet. 2014;384(9948):1109-1117.

[27] Chen DS, Mellman I. Oncology Meets Immunology: The Cancer-Immunity Cycle. Immunity. 2013;39(1):1-10.

[28] Vilgelm AE, Johnson DB, Richmond A. Combinatorial approach to cancer immunotherapy: strength in numbers. J Leukoc Biol. 2016;100:149-160. 
[29] Shindo Y, Yoshimura K, Kuramasu A, Watanabe Y, Ito H, Kondo T, Oga A, Ito H, Yoshino S, Hazama S, Tamada K, Yagita H, Oka M. Combination Immunotherapy with 4-1BB Activation and PD-1 Blockade Enhances Antitumor Efficacy in a Mouse Model of Subcutaneous Tumor. Anticancer Res. 2015;136:129-136.

[30] Chen S, Lee L-F, Fisher TS, et al. Combination of 4-1BB Agonist and PD-1 Antagonist Promotes Antitumor Effector/Memory CD8 T Cells in a Poorly Immunogenic Tumor Model. Cancer Immunol Res. 2014;3(2):149-160.

[31] Buchan SL, Manzo T, Flutter B, et al. OX40- and CD27-mediated costimulation synergizes with anti-PD-L1 blockade by forcing exhausted CD8+ T cells to exit quiescence. J Immunol. 2015;194(1):125-33.

[32] Wrzesinski C, Paulos CM, Kaiser A, et al. Increased intensity lymphodepletion enhances tumor treatment efficacy of adoptively transferred tumor-specific T cells. $J$ Immunother. 2010;33(1):1-7.

[33] Li B, Vanroey M, Wang C, Chen THT, Korman A, Jooss K. Anti-programmed death-1 synergizes with granulocyte macrophage colony-stimulating factor-secreting tumor cell immunotherapy providing therapeutic benefit to mice with established tumors. Clin Cancer Res. 2009;15(5):1623-1634. 